\title{
Parental Monitoring Scale (PMS): A tool to measure parental monitoring perception of schooling adolescents (14 -16 years) in Sri Lanka
}

\author{
Gayani K. P. De Silva ${ }^{1}$, Devani S. Dissanayake ${ }^{2}$ \\ ${ }^{1}$ Family Health Bureau, Ministry of health, Sri Lanka \\ ${ }^{2}$ Department of Community Medicine, Faculty of Medicine, University of Peradeniya, Sri Lanka \\ DOI: 10.29322/IJSRP.12.01.2022.p12148 \\ http://dx.doi.org/10.29322/IJSRP.12.01.2022.p12148
}

\begin{abstract}
Parental monitoring is a culturally sensitive concept which is shown to affect the wellbeing of adolescents. Adolescents of school going age need to be monitored to prevent them experiencing risk behaviors as they are spending much time outside houses, not in the presence of their parents and with friends or strangers. Since there was no tool to assess the perception of parental monitoring among school going adolescents (14 -16 years), the objective of the study was to adapt, translate and validate an instrument (PMS - Adolescent - Sinhala version) to assess parental monitoring as perceived by the school going adolescents (14-16 years) in Sri Lanka.
\end{abstract}

\section{Methods:}

Culturally adapted, translated to Sinhala language and pre-tested PMS was administered to a convenient sample of 556 school going adolescents (14-16 years) in Denuwara Education Zone. Construct validity was established through exploratory factor analysis and multi trait scaling analysis using SPSS version 22 and confirmatory factor analysis using LISREL 9.2. Internal consistency and test-retest methods were used to assess reliability.

\section{Results:}

Exploratory factor analysis revealed culturally adapted PMS as a tool with a four-factor structure with 23 items. Confirmatory factor analysis confirmed that model construct with a combination of acceptable absolute, relative and parsimony fit indices reaching desired threshold values (SRMR of 0.063, CFI $<0.9$ and RMSEA with 0.061). A Cronbach's alpha value exceeding 0.8 within the four subscales assured high internal consistency. The test-retest reliability was also high $(\mathrm{p}<0.001)$.

\section{Conclusion:}

The Sinhala version of the 23-item PMS is a valid and a reliable instrument to assess the level of parental monitoring perception among school going adolescents in Sri Lanka.

Index Terms- Adolescents, Parental monitoring Reliability, Validity

\section{INTRODUCTION}

Parental monitoring has been defined as 'parents' knowledge about their adolescents' whereabouts, activities, and companions and the sources which parents use to gain that information" [1]. This behavioral construct explains a composite of parenting practice variables including parental awareness, communication with adolescents, parental concern, supervision by parents including regularly checking on something, being knowledgeable about and tracking of adolescent behaviors in a broader setting. When adolescents perceive their parents make a habit of knowing about their activities, companions and whereabouts they try to act by setting clear expectations for acceptable behaviors reducing their risk behavior involvement [2]. Globally, parental monitoring has been identified as an important parenting practice which adversely affected adolescent's wellbeing and health. Continuous parental monitoring is important as adolescents are expecting their independence and as they are spending more time outside homes doing different activities with friends in the society as parents need to be alert about their adolescent children [3], [4]. Parental monitoring perception is an important assessment to use in order to prevent or minimize adolescent involvement with the risk behaviors. Therefore, it is evident that it is essential to identify the adolescents' perception about their parents' monitoring behavior.

Since there is no comprehensive tool to assess parental monitoring among school going adolescents in sri Lankan context, it was identified the importance of having a tool to assess the level of parental monitoring as perceived by adolescents. Therefore, adolescent version of the Parental Monitoring Scale (PMS) by Stattin and Kerr, was culturally adapted and validated during this study. This scale has been widely used among adolescents in the age group of 14-16 years in many different cultural settings to assess parental monitoring perception.

\section{METHODS}

\section{Structure of the original PMS}

The 24-item PMS is a self-administered questionnaire (SAQ) with ten items assessing parental knowledge and five items in each subscale assessing the sources of parental monitoring, adolescent disclosure (AD), parental solicitation (PS) and parental control (PC). A five-point rating scale is used to assess the frequency in which the respondents experience feelings related to each dimension and this rating scale ranges from 1 (never) to 5 (almost always). According to the scores of each dimension, the 
high scores of each scale as well as the total scale are indicative of high parental monitoring perception.

\section{Translation and pre-testing of the PMS}

PMS was translated to Sinhala by using the forwardbackward translation method [5]. Two bilingual translators, who are fluent in Sinhala and English, independently translated the questionnaire into Sinhala while ensuring semantic equivalence, conceptual equivalence, and normative equivalence. Any discrepancies and ambiguities between the translated versions and any deficiencies compared to the original English version were resolved by consensus. The synthesized forward translated version was agreed upon for the backward translation. Two sworn language translators, who were totally blind to the original English version of the PMS, independently translated the synthesized forward translation of PMS back into English, without referring to the original version.

Pre-testing of the synthesized forward translation of the PMS was conducted among a sample of 30 grade nine and ten students who were studying in schools outside the study setting. This sample consisted of both male and female students studying in different school categories. In a subsequent structured interview, the clarity in acceptability, understanding, and comprehension of items and feasibility of using the questionnaire with the average time taken by the students to complete the questionnaire were assessed. None of the items of the questionnaire was claimed to be difficult to understand.

\section{Appraising the judgmental validity of the PMS}

The face, content, and consensual validity were assessed using a modified Delphi technique. A multi-disciplinary panel of experts in the fields of community physicians, psychiatrist, psychologist and sociologist assessed each item on its relevance, appropriateness, and acceptability in the local context for assessing parental monitoring perception among school going adolescents (14 -16years) based on a rating scale from 1 (strong disagreement) to 9 (strong agreement). In addition to rating of each item, the panelists were asked to make additional remarks related to the phrasing of items and to indicate any additional specific items that they think are relevant in relation to parental monitoring in Sri Lanka. At the end of this iterative process, all the experts were of opinion that all original items are relevant in assessing parental monitoring of adolescents with a median score more than 7 for all the aspects. The words of the item number 7 , 9, 14, 16, 18 and 19 were changed according to the Sri Lankan culture. Item, about assessment on parental knowledge on use of mobile phones, internet and social media networks, which they thought as important in assessing parental monitoring acts related to adolescents of Sri Lankan school setting was added by the experts in panel. Permission to include the relevant item was obtained by the original author. Finally, questionnaire with 25 items in the translated Sinhala version of PMS was forwarded for assessment of construct validity.

\section{Assessment of Construct validity}

Study design and setting - A school-based, cross-sectional validation study was conducted in Mawanella education division, Sabaragamuwa province in Sri Lanka from October to November 2019 to assess construct validity of PMS in two steps. Six schools were purposely selected from the school list of Sinhala medium government schools of type $1 \mathrm{AB}$, type $1 \mathrm{C}$ and Type 2 categories from Mawanella education division to include all three categories of school types (two schools from each category). All the students from grade 9 and 10 of the randomly selected schools representing both male and female students were recruited for the study components provided confirming the inclusion criteria.

Study participants - Randomly selected three schools out of six were recruited for the first step of the study component which the data was collected for the PCA. The total number of students participated in this step was 278 and the response rate was $100.0 \%$. Of study sample $54.3 . \%(n=151)$ were girls. The mean age was 15.3 years ( $\mathrm{SD}=0.43$ years). Data collection was done using the Sinhala translated and pretested draft of PMS with 25 items. Another 278 students from grade 9 and 10 of the randomly selected other three schools out of six were recruited for the second step of the study component who were not included in PCA. The response rate was $100.0 \%$ and $50.0 \%(n=141)$ of the sample were girls. The mean age was 15.7 years ( $\mathrm{SD}=0.42$ years). Data was collected using the draft PMS with 23 items obtained following the Exploratory factor analysis and Multi trait scaling analysis.

Data analysis - Data were analyzed by using the Statistical Package for Social Sciences (SPSS) version 22.0. Preparatory data analysis showed that there were no violations of the assumptions related to the data analytic techniques. Given that the participants to variables ratio was 11.4 , the sample size was adequate for factor analysis [6]. Even though there were few items that showed nonnormal distribution of data, it is a ubiquitous phenomenon in psychological assessment research.

Multi-trait scaling analysis - Item-scale correlations were analyzed and item-convergent and item-discriminant validity were assessed. A stringent criterion of correlation of 0.40 or greater between an item and its own subscale was considered as a success for assessing item-convergent validity. Furthermore, in assessing item-discriminant validity, items that correlated significantly higher (more than 1.96 standard errors) with its own subscale than with the other two subscales, were considered as scaling successes.

Exploratory factor analysis (EFA) - EFA was conducted by Principal Component Analysis (PCA) with Promax rotation. Kaiser's criterion/ eigenvalue and scree plot were used to decide the number of factors to retain. The factors that lead to a meaningful interpretation and theoretical sense were ultimately selected.

Confirmatory factor analysis (CFA) - Considering the nonnormal distribution of the items in the PMS and according to the recommendations offered in LISREL 9.2, Robust Maximum Likelihood (RML) estimation method was used in the CFA. Assessment of the appropriateness of the model was done based on several fit indices including absolute fit indices, relative fit 
indices and parsimony fit indices. Satorra-Bentler scaled chisquare test, Root Mean Square Error of Approximation (RMSEA), Goodness-of-Fit Index (GFI), Adjusted Goodness-of-Fit Index (AGFI), and Standardized Root Mean Square Residual (SRMR) were used as the absolute fit indices. Comparative Fit Index (CFI) and Non-Normed Fit Index (NNFI) were used as the relative fit indices, while Parsimony Goodness-of-Fit Index (PGFI) and Parsimonious Normed Fit Index (PNFI) were used as the parsimony fit indices. Judgements on how well the model fits data were made based on RMSEA < 0.05, SRMR 0.9 and NNFI >0.9 [7].

\section{Assessment of reliability}

In order to assess the reliability or the consistency of information gathered by the Sinhala version of PMS, two methods, viz., internal consistency and test-retest reliability were employed. Internal consistency was assessed in the study using the Chronbach's Alpha for each subscale of PMS, where it is estimated that values higher than 0.7 are satisfactory [8], [9]. Testretest reliability was assessed by administering the Sinhala version of PMS after a gap of two weeks in a sub-sample of participants enrolled in the study. Value of 0.7 or above for the Spearman's $r$ correlation coefficient was considered as having at least a moderate correlation between the scores [10].

\section{RESULTS}

\section{Cultural adaptation and appraising judgmental validity of PMS}

During cultural adaptation process the original items were modified according to the cultural relevance of the items and one new item was included by the expert panel. Item number 7, 9, 14 and 18 the word and the concept of "at night" was changed as "during evenings until night" and in item number 16 and 19, the word and the concept of "Saturday evening" was changed as "holiday evenings". This was discussed during the Delphi rounds in cultural adaptation process "as being out-side the houses without parents at night" is not accepted and not practiced among children in Sri Lankan culture and "holidays" were considered more commonly than "Saturdays". Furthermore, one new item was included to the questionnaire during the Delphi process, and it was confirmed to be included during judgmental validation process.

During assessment of consensus validity, item number six and seven obtained median scores at the range of (4-7) during all three rounds as rated by the members in the panel. Further these two items were commented as having ambiguity with other items in the scale. All the other items had obtained rating scores in the range of (7-9). Based on the compiled rating scores and comments, it was decided to include all items of the original scale with added new one item in the synthesized forward translation of final PMS with suggested modifications and considered for the validation study.

\section{Multi trait scaling analysis}

Results of the MTSA conducted on PMS validation study are summarized in Table 3. Item convergent validity was supported if an item correlation was 0.4 or more with the scale it is hypothesized to represent. Item convergent coefficients are reasonably large, thus the items representing the four traits are internally constant. All the item correlations were more than 0.4 and met with the criteria, hence all items' item convergence was confirmed. Item discrimination was supported if the correlation between an item and the subscale that it is hypothesized to measure was significantly larger (more than 1.96 standard errors) than the correlations of that item with other subscales. Except for the items sixth and seventh, all item-scale correlations for other items emerged as scaling successes. Hence, according to the MTSA, except for the items sixth and seventh, item-convergent validity and item-discriminant validity were confirmed for other 23 items in PMS.

\section{Exploratory factor analysis}

Items sixth and seventh were found to have poor psychometric properties in judgmental validity, item-convergent validity, and item-discriminant validity. Deletion of those items from the subscale also improved the internal consistency. Hence, both 25 item PMS and 23 items of the PMS (item $6^{\text {th }}$ and $7^{\text {th }}$ deleted) were subjected to PCA. The Kaiser-Meyer-Olkin Measure were 0.828 and 0.855 respectively and Bartlett's test of sphericity reached statistical significance $(\mathrm{p}<0.001)$ for both versions. The number of factors was decided depending on the Eigen value during factor extraction. A factor was considered relevant if the given Eigen value exceeded 1.0. Extraction was done depending on the eigen value of $>1$. Initially six factors were extracted which cumulatively explaining $63.9 \%$ of the total variance and following deletion of item $6^{\text {th }}$ and $7^{\text {th }}$ four factors were extracted cumulatively explaining with $70.1 \%$ of the total variance which had eigenvalues more than 1.0 .

Due to the large discrepancy in the interpretations between the factor's structures observed in the eigenvalues versus scree plot, it was difficult to understand the underlying latent construct. The ensuing process required an appraisal of individual items for possible removal. A criterion for item deletion was determined by the values of the item loadings and cross-loadings on the factors were done during factor rotation. Rotations generally result in a more interpretable solution and one that is more likely to generalize to other samples from the same population [11].

However, for both versions, the component matrix in PCA with unrotated loadings revealed a number of items of PMS loading on four factors with values greater than 0.3 . Furthermore, the pattern matrix generated in PCA using direct ProMax rotation revealed that seven items had loading values more than 0.3 . Based on this collective evidence, it was decided to 'force' a four-factor solution for further investigation. Furthermore, for 23 items version of PMS, the scree plot showed a clear break after the fourth factor as well which seemed to be more appropriate. Therefore, four-factor solution was adopted for the current study. Table 4 shows the total variance explained in PCA after removing Item $6^{\text {th }}$ and $7^{\text {th }}$ in PM. In the 23-item PMS version, the four-factor solution explained a total of $70.1 \%$ of the variance, with factor one contributing $34.4 \%$,

This publication is licensed under Creative Commons Attribution CC BY.

http://dx.doi.org/10.29322/IJSRP.12.01.2022.p12148

wWw.ijsrp.org 
factor two contributing $11.3 \%$, factor three contributing $9.3 \%$ and factor four contributing $8.99 \%$. 
Table 3: Item-Scale Correlations of PMS, item-Convergent and item-Discriminant Validity $(\mathrm{n}=278)$

\begin{tabular}{|c|c|c|c|c|c|c|c|}
\hline Item & PKS & ADS & PCS & PSS & SE & -1.96 SE Cut-off value & Scaling Success \\
\hline PK1 & 0.865 & 0.694 & 0.652 & 0.484 & 0.045 & 0.697 & success \\
\hline PK2 & 0.885 & 0.470 & 0.519 & 0.388 & 0.047 & 0.672 & success \\
\hline PK3 & 0.865 & 0.516 & 0.480 & 0.528 & 0,045 & 0.690 & success \\
\hline PK4 & 0.875 & 0.552 & 0.471 & 0.446 & 0.046 & 0.704 & success \\
\hline PK5 & 0.888 & 0.244 & 0.222 & 0.168 & 0.043 & 0.698 & success \\
\hline PK6 & 0.663 & 0.649 & 0.496 & 0.382 & 0.064 & 0.231 & not success \\
\hline PK7 & 0.686 & 0.594 & 0.439 & 0.369 & 0.074 & 0.261 & not success \\
\hline PK8 & 0.817 & 0.515 & 0.512 & 0.530 & 0.034 & 0.667 & success \\
\hline PK9 & 0.806 & 0.456 & 0.472 & 0.221 & 0.045 & 0.723 & success \\
\hline PK10 & 0.861 & 0.579 & 0.595 & 0.392 & 0.024 & 0.731 & success \\
\hline AD11 & 0.569 & 0.794 & 0.306 & 0.268 & 0.034 & 0.761 & success \\
\hline AD12 & 0.539 & 0.785 & 0.353 & 0.323 & 0.036 & 0.727 & success \\
\hline AD13 & 0.655 & 0.852 & 0.615 & 0.471 & 0.043 & 0.610 & success \\
\hline AD14 & 0.600 & 0.781 & 0.589 & 0.580 & 0.042 & 0.764 & success \\
\hline AD15 & 0.502 & 0.879 & 0.545 & 0.445 & 0.026 & 0.699 & success \\
\hline PC16 & 0.526 & 0.570 & 0.831 & 0.320 & 0.028 & 0.624 & success \\
\hline PC17 & 0.552 & 0.662 & 0.803 & 0.417 & 0.045 & 0.723 & success \\
\hline PCS18 & 0.520 & 0.464 & 0.800 & 0.522 & 0.041 & 0.721 & success \\
\hline PCS19 & 0.363 & 0.405 & 0.857 & 0.602 & 0.043 & 0.659 & success \\
\hline PCS20 & 0.371 & 0.493 & 0.816 & 0.315 & 0.034 & 0.711 & success \\
\hline PSS21 & 0.519 & 0.407 & 0.460 & 0.851 & 0.023 & 0.663 & success \\
\hline PSS22 & 0.423 & 0.492 & 0.572 & 0.860 & 0.021 & 0.621 & success \\
\hline PSS23 & 0.383 & 0.477 & 0.464 & 0.801 & 0.028 & 0.762 & success \\
\hline PSS24 & 0.512 & 0.387 & 0.471 & 0.789 & 0.041 & 0.722 & success \\
\hline PSS25 & 0.443 & 0.651 & 0.466 & 0.798 & 0.043 & 0.716 & success \\
\hline
\end{tabular}


Table 4: Total Variance Explained in PCA After Removing Item no $6^{\text {th }}$ and $7^{\text {th }}$ in PMS

\begin{tabular}{|c|c|c|c|}
\hline \multirow[b]{2}{*}{$\begin{array}{l}\text { Componen } \\
\text { t }\end{array}$} & \multicolumn{2}{|c|}{ Initial Eigenvalues } & \multirow[b]{2}{*}{ Cumulative \% } \\
\hline & Total & $\%$ of Variance & \\
\hline 1 & 7.920 & 34.435 & 44.435 \\
\hline 2 & 2.608 & 11.338 & 55.773 \\
\hline 3 & 2.136 & 9.288 & 65.061 \\
\hline 4 & 2.069 & 8.996 & 70.128 \\
\hline 5 & 0.991 & 4.310 & 71.368 \\
\hline 6 & 0.906 & 3.940 & 72.308 \\
\hline 7 & 0.831 & 3.614 & 75.921 \\
\hline 8 & 0.730 & 3.174 & 79.095 \\
\hline 9 & 0.621 & 2.700 & 81.795 \\
\hline 10 & 0.606 & 2.634 & 84.429 \\
\hline 11 & 0.547 & 2.378 & 86.807 \\
\hline 12 & 0.447 & 1.945 & 88.751 \\
\hline 13 & 0.397 & 1.724 & 90.475 \\
\hline 14 & 0.353 & 1.534 & 92.010 \\
\hline 15 & 0.326 & 1.416 & 93.426 \\
\hline 16 & 0.268 & 1.165 & 94.591 \\
\hline 17 & 0.244 & 1.061 & 95.652 \\
\hline 18 & 0.239 & 1.039 & 96.691 \\
\hline 19 & 0.213 & 0.928 & 97.619 \\
\hline 20 & 0.176 & 0.765 & 98.384 \\
\hline 21 & 0.160 & 0.697 & 99.081 \\
\hline 22 & 0.123 & 0.536 & 99.616 \\
\hline 23 & 0.088 & 0.384 & 100.000 \\
\hline
\end{tabular}

Even though both Varimax rotation and ProMax rotation was used, it was decided to report statistics related to Promax rotation with Kaiser Normalization was conducted as the factors were strongly correlated $(>0.3)$ [12] and given the subjective nature of participants' perceptions and experiences. Table 5 shows the rotated component matrix / pattern and structure matrix for PCA after item $6^{\text {th }}$ and $7^{\text {th }}$ were dropped with Promax rotation of fourfactor solution of 23-item PMS.
Table 5: Rotated Component Matrix of Items of the PMS, after Item no $6^{\text {th }}$ and 7 th were Dropped

\begin{tabular}{|c|c|c|c|c|}
\hline Item & 1 & 2 & 3 & 4 \\
\hline PKS 1 & & & & 0.663 \\
\hline PKS 2 & & & & 0.728 \\
\hline PKS 3 & & & & 0.512 \\
\hline PKS 4 & & & & 0.775 \\
\hline PKS 5 & & & & 0.774 \\
\hline PKS 8 & & & & 0.506 \\
\hline PKS 9 & & & & 0.599 \\
\hline PKS 10 & & & & 0.532 \\
\hline ADS 1 & & & 0.874 & \\
\hline ADS 2 & & & 0.761 & \\
\hline ADS 3 & & & 0.812 & \\
\hline ADS 4 & & & 0.806 & \\
\hline ADS 5 & & & 0.862 & \\
\hline PCS 1 & & 0.873 & & \\
\hline PCS 2 & & 0.768 & & \\
\hline PCS 3 & & 0.881 & & \\
\hline PCS 4 & & 0.896 & & \\
\hline PCS 5 & & 0.874 & & \\
\hline PSS 1 & 0.860 & & & \\
\hline PSS 2 & 0.878 & & & \\
\hline PSS 3 & 0.884 & & & \\
\hline PSS 4 & 0.776 & & & \\
\hline PSS 5 & 0.728 & & & \\
\hline
\end{tabular}

Extraction Method: Principal Component Analysis / Rotation Method: Promax with Kaiser Normalization / Rotation converged in 4 iterations

The interpretations of the four-factor solution of the PMS with items $6^{\text {th }}$ and $7^{\text {th }}$ deleted, was consistent with previous research on the PMS with subscale items of parental control(PC), parental solicitation(PS), adolescent disclosure(AD) and parental knowledge(PK) loading strongly on four different factors. The four factors identified were coincides with the original scales as well. So, the final numbers of items were 23 in four factor structure was used for further analysis. 


\section{CFA}

\section{Assessment of data set for comparability for confirmatory factor analysis}

The sample size was 278 for 23 observed variables. The ratio observations to variables were approximately 11.1:1. Normality of data was assessed by Kolmogorv - Smirnov and Shapiro - Wilk test of normality which were statistically significant $(<0.05)$ confirming non normality of data. Histograms drawn for each item showed a non-normal distribution of data. The standardized skewness and kurtosis were calculated. These ratios were greater than 1.96 had a $p$ value of less than 0.05 , indicating a significant skew or kurtosis. Data was checked for outliers using the boxplot for univariate outliers. Although there were few univariate outliers, they were included in the dataset as it was decided that they were important to be included following the opinion of a panel of experts. Bivariate correlations between items were examined. The highest correlation observed between the two items was 0.706 , indicating that none of the two items were highly or perfectly correlated. Linearity of the data set was assessed though inspection of the bivariate scatter plots only for random item samples as it is practically difficult to conduct the inspection and present of all combinations. Examined scatter plots showed a linear relationship.

\section{Assessment of construct validity through CFA}

In relation to factorial validity, as the four-factor model of PMS is established through PCA in this study during previous analysis, CFA was employed to assess in a different sample the extent to which underlying four-factor model was replicated in the observed data. Linear Structural Relations (LISREL) version 9.1 was used for the analysis. Since the distribution of responses obtained for each item did not confirm to a normal distribution, Robust Maximum Likelihood (RML) method was carried out for CFA to overcome the issue of skewed distribution of data [13]. Several models with different combinations of factor structures were evaluated using RML estimation method.

The structure of the PMS was confirmed based on a variety of fit indices, including absolute fit indices, relative fit indices and parsimony fit indices. Satorra-Bentler scaled chi-square test, Goodness-of-Fit Index (GFI) and Standardised Root Mean Square Residual (SRMR) were used as the absolute fit indices. Comparative Fit Index (CFI) and Non-Normed Fit Index (NNFI) were used as the relative fit indices, while Root Mean Square Error of Approximation (RMSEA) was used as the parsimony fit indices. Based on the recommendations of $\mathrm{Hu} \&$ Bentler, 1999, model fit for the sample was evaluated using a combination of above fit indices using according to the literature reviewed cut-off scores. Specifically, good fit was indicated by RMSEA $\leq 0.06$, SRMR $\leq 0.08$, GIF $>0.90$ and CFI $\geq 0.90$ and NNFI, $\geq 0.90$. Following models were assessed during analysis.

a) One-factor model: All 23 items of PMS were loaded on to one latent factor. b) Two-factor model: Items measuring PK (eight items) and other 15 items were loaded on to one latent factor named as "sources of knowledge".

c) Three-factor model: Items measuring PK (eight items) were loaded on to one latent factor. Ten items in the PS and PC scales' items were loaded on to one latent factor named as "parental behaviour" while the five items were loaded to AD factor.

d) Four factor model - Items measuring PK, AD, PS and PC which the factors identified from EFA were loaded on to four separate latent factors.

The summary of model fit statistics of the three models described above is given in table 6 .

Table 6: Model Fit Statistics of the One Factor, Two Factor, Three Factor and four Factor Models of PMS

\begin{tabular}{rrrrrr}
\hline Model Absolute fit indices & $\begin{array}{c}\text { Comparative } \\
\text { fit indices }\end{array}$ & $\begin{array}{c}\text { Parsimo- } \\
\text { ny } \\
\text { correlate } \\
- \text { on }\end{array}$ \\
\hline$\chi 2$ & GFI & $\begin{array}{r}\text { SRM } \\
\text { R }\end{array}$ & $\begin{array}{r}\text { NN } \\
\text { FI }\end{array}$ & CFI & RMSEA \\
& & & &
\end{tabular}

\begin{tabular}{|c|c|c|c|c|c|c|}
\hline $\begin{array}{l}\text { One } \\
\text { factor }\end{array}$ & $\begin{array}{r}280.37 \\
\mathrm{df}=196 \\
0.000\end{array}$ & 0.57 & 0.093 & 0.83 & 0.84 & 0.131 \\
\hline $\begin{array}{l}\text { Two } \\
\text { factors }\end{array}$ & $\begin{array}{r}251.68 \\
\mathrm{df}=192 \\
0.000\end{array}$ & 0.6 & 0.086 & 0.88 & 0.88 & 0.11 \\
\hline $\begin{array}{l}\text { Three } \\
\text { factors }\end{array}$ & $\begin{array}{r}272 \\
\mathrm{df}=185 \\
0.000\end{array}$ & 0.81 & 0.082 & 0.94 & 0.94 & 0.086 \\
\hline $\begin{array}{l}\text { Four } \\
\text { factors }\end{array}$ & $\begin{array}{r}258 \\
\mathrm{df}=164 \\
0.000\end{array}$ & 0.92 & 0.063 & 0.96 & 0.92 & 0.061 \\
\hline \multicolumn{7}{|c|}{$\begin{array}{l}\chi^{2} \text { chi square test }-(p<0.05 \text { desired }) \\
\text { GFI - Goodness of fit index }(>0.9 \text { desired }) \\
\text { SRMR - Standardized root mean square residual }(<0.08 \text { desired }) \\
\text { NNFI - Non normed fit index }(>0.9 \text { desired }) \\
\text { CFI-Comparative fit index }(>0.9 \text { desired }) \\
\text { RMSEA - Root mean square error of approximation }(<0.05 \\
\text { desired) }\end{array}$} \\
\hline
\end{tabular}

Finally, the fourth model with four factors which was identified in EFA revealed a significant better fit for the fourth-factor model $\left(\chi^{2}=258 ; \quad \mathrm{df}=164 ; \quad \mathrm{p}=0.000 ; \quad \mathrm{RMSEA}=0.061 ; \quad \mathrm{SRMR}=0.063\right.$; $\mathrm{CFI}=0.921)$. This model showed the best model fit statistics out of 
all the models tested. All the factor loadings of this model were statistically significant $(\mathrm{p}<0.05)$. Furthermore, all the items had factor loadings larger than 0.6 from its own latent factor.

\section{Assessment of reliability of PMS}

To determine whether the above 23 items selected from the EFA, represented an internally consistent measure, the PI computed an overall Cronbach alpha $(\alpha)$ coefficient for each subscale of the PMS. Validated PMS consisted of eight items measuring PK subscale and five items for each subscale measuring AD subscale, PC subscale and PS subscale. Impact of each item on the related subscale was assessed by computing Cronbach's $\alpha$ when the respective item is deleted. None of the items in PS, PC and AD subscales improved the overall Cronbach's $\alpha$ value; however, deletion of item $6^{\text {th }}$ and $7^{\text {th }}$ improved the Cronbach's $\alpha$ value from 0.696 to 0.867 in PK subscale. Hence, those items were removed from the PMS and the reliability was re-assessed for internal consistency and all four subscales show high internal consistency with Cronbach's $\alpha$ coefficient values of $0.867,0.819,0.843$ and 0.898 for PK, AD, PC and PS subscales respectively which were satisfied.

There were strong positive correlations for each of the four subscales in test-retest reliability assessment. The correlation coefficient for the total scale was 0.857 which indicates that there is good inter-rater reliability. The correlation coefficients were statistically significant at $\mathrm{p}<0.001$.

\section{DISCUSSION}

The present study was designed to culturally adapt and validate the Sinhala version of the PMS initiating addressing an important research vacuum on parental monitoring perception research in school students in the South Asian context. A common and globally used [15][19] factor structure of PMS was consistent with the operational definition of parental monitoring used in the present study as well. PMS is a self-reported tool which is administrable assure the confidentiality of responses to the participants. The average time spent to administer the instrument was 15 -20 minutes which was considered satisfactory for a school-based study and could easily be used since the entire sample population in the present study was of a similar level of education who could easily understand this SAQ. A crosssectional design and the sample size of the study deemed appropriate with the study objective. Adolescent perception is a culturally diverse generic measure of parental monitoring. It is important to select a sample from a study population with varying perceptions, different social and geographical conditions to test a scales' validity. So, study setting was selected in view of identifying a different study population of the same age group with diverse cultural background. The present study utilized a carefully designed method whereby validity, reliability and acceptability of the different aspects that are assessed by the tool were evaluated using different validation methods.

Outcome responses measured from the scale may vary on the basis of country, culture and language. To overcome the effect of these changes, a proper translation process along with adequate modifications should be followed to ensure equivalence [20]. The cultural adaptation was done in the present study with a qualitative research method, modified Delphi method, which enables obtaining views of important stakeholders. It became evident that the instrument lacked assessing an area related to parental knowledge on the internet and social media use of adolescents which was discussed during the Delphi process in cultural adaptation as it may be relevant in the current socioeconomic setting. Through discussions and feedback from experts during the Delphi process, it was decided to add one item to the instrument. The additional item was placed after the original items and thus did not alter the instrument's original structure. The forward and backward translation method was employed in the present study ensuring its' semantic equivalence, conceptual equivalence and normative equivalence. All these were achieved by conducting this in involvement with both language experts and technical experts. A maximum effort was taken to maintain content validity during the translation process.

A multi-disciplinary panel of experts judgmentally validated the tool during the modified Delphi method by scoring each item for its relevance in assessing parental monitoring among school-going adolescents of 14-16 years, appropriateness of wordings that were used during translation and acceptability of the items in the local context in assessing parental monitoring. This rigorous process of qualitative and quantitative methods to assess the extent of agreement (consensus measurement) and to resolve disagreements (consensus development) in a confidential environment ensured a valid and acceptable procedure for cultural adaptation. Pre-testing of the PMS was done in a separate district in a randomly selected sample of 30 schooling adolescents of the same age including both males and females.

In order to estimate the relative contribution of item variance, more than one method must be employed in a validation process (Arafat et al., 2016). MTSA was used in the present study to assess the hypothesized scale structure of PMS as the primary step in analyzing whether the set of items in PMS can be suitably combined into a summated rating scale. In MTSA, except for the item $6^{\text {th }}$ and $7^{\text {th }}$, item-convergent validity and item-discriminant validity were confirmed for other 23 items in the PMS. The ambivalent nature of those two items may have resulted in not having satisfactory item-convergent and item-discriminant validity in MTSA.

Linked to the constructive assumptions, Stattin and Kerr (2000) proposed a set of subscales, parental knowledge scale, adolescent disclosure scale, parental control scale and parental solicitation scale for the assessment of parental monitoring of adolescents. Researchers have been using these subscales by adapting them either as a whole assessment tool or as selected sets of the subscales (one or more subscale in combination with) in assessing parental monitoring depending on their research aim and according to their operational definitions [21] [23]. The present study conceptualized parental monitoring of adolescents as the perception among adolescents on "knowledge of parents and sources of that knowledge about whereabouts, activities and companions". Thus, all four subscales have been used in the study as PMS for the assessment of parental monitoring of adolescents. As the factor structure is not an established construct for the 
intended context, EFA was conducted before construct validation by CFA.

Even though PMS with four-factor structure has been confirmed in a large number of studies [24] there are limited number studies that explored its' factor validity. It has been further highlighted that EFA analysis provides the best option for identification of the constructs, especially when the constructs cannot be hypothesized in the using context [25] and in exploring factor structures without strong hypotheses [24]. As this parental monitoring concept is hypothesized for the first time in the present study context, EFA was performed to explore the underlying latent factors in PMS. This evidence coupled with the fact that PMS is a relatively novel measure in the South-East Asian context, EFA was employed in combination with the CFA.

The same procedure has been adopted by Fernando (2019), for validation of a tool to assess behavior related stigma among key populations in Sri Lanka. After reviewing the pattern matrix, it was decided to remove item numbers sixth and seventh due to their loadings on to a different two factors within the specified criteria for removal (the difference between factor loadings was $<0.15$ ). Since sixth and seventh items showed unsatisfactory results at multi-trait-scaling analysis and since they were cross loaded during EFA, both those items were deleted, and other items were re-rotated and found that the items were loading well to four factors which were further supported by the scree plot as well. Finally, the original 22 items with a newly added item were loaded into four factors as a result during EFA in the study.

CFA is a multivariate statistical method used in research to assess construct satisfaction. It needs a strong hypothetical construct underlying the measurement model before analyzing data $(\mathrm{Li}$, 2014). Since there are no consciences to what type of model fit indices are to be used in assessing the model fit, a combination of absolute and relative fit indices was used in the present study. The result of the construct validation finally showed that the fourdimensional structure used in the original PMS as well as explored from the EFA showed improved model fit statistics to the accepted levels of indices than other tested factor models. Similarly, this four-factor model has been confirmed by CFA in a study done in Italy by Bacchini et al., (2011). The same construct has been further confirmed by evaluation of convergent validity using correlation assessment with another parental monitoring scale. They have further established the construct validity by concurrent and predictive validity assessment methods using longitudinal assessment of aggression behavior instrument [26]. The factorial validity of these four subscales of PMS had been assessed on a large sample of Italian adolescents [27]. During analysis, they have evaluated the fit of each subscale separately as well as the fit of the theorized model of all four subscales. This study concluded that this scale has an acceptable factorial validity in the assessment of parental monitoring. Even though, the results came as a fourfactor structure in the present study, in a study done in a Swedish sample aiming validation of this PMS has shown a five-factor structure with PK, PS, PC' AD and adolescent secrecy [28]. These latter two subscales were identified as one in the in the present study, as well as in the Swedish sample by Stattin \& Kerr in 2000, giving an acceptable model fit. A comparable result has been obtained from another study done subsequently in an American sample of adolescents where marginally accepted fit indices were obtained for this secrecy subscale [29]. In both instances' secrecy was obtained as a different subscale, have used a smaller sample size than the present study.

The reliability of the PMS was assessed using internal consistency and test-retest reliability and those revealed reliability estimates were consistent with the reliability estimates obtained in the Swedish version of PMS, which was $0.85,0.81,0,7$ and 0.78 for PK, AD, PS and PC subscales respectively [30], [31].

The present study revealed that the test-retest correlation coefficient was high for the four subscales of PMS after two weeks following the initial administration and was statistically significant $(\mathrm{r}=0.857, \mathrm{P}<0.001)$. The repeatability of a test is considered as an equally important measure to obtain valid and consistent estimates from a test. The test-retest reliability of PMS to detect parental monitoring perception showed good results indicating PMS ability to produce consistent results with repeated use.

This study has some limitations. Since there was no gold standard to measure parental monitoring perception of adolescents, the criterion validity of PMS was not assessed. Given that the results generated from factor analysis are often sample specific (MacCallum, et al 1999) generalizability of the present study findings to other populations should be done with caution, considering the variations in educational and cultural contexts. Furthermore, selecting the six Sinhala medium government schools considering the logistic feasibility without using a probability sampling technique is another study limitation, which affects the generalizability of the study findings.

\section{CONCLUSION}

The Sinhala translated version of the 23-item Parental Monitoring Scale with four factors is a valid and reliable instrument to measure parental monitoring perception of school going adolescents of the age group of 14-16 years in Sri Lanka.

\section{APPENDIX}

Appendixes, if needed, appear before the acknowledgment.

\section{ACKNOWLEDGMENT}

I acknowledge the study population who participated in this study and the experts in psychology, public health and sociology who gave valuable inputs to make this study a success.

Declaration of interest: I declare that there is no conflict of interest.

\section{REFERENCES}

[1] M. Kerr and H. Stattin, "What parents know, how they know it, and several forms of adolescent adjustment: further support for a reinterpretation of monitoring.," Developmental psychology, 2000, doi: 10.1037/0012-1649.36.3.366

[2] L. Hayes, "Parental Monitoring of Adolescent Free Time : a Theoretical Model of Parent-Adolescent Interactions," no. July, 2004 
[3] M. Kerr, H. Stattin, and M. Özdemir, "Perceived parenting style and adolescent adjustment: Revisiting directions of effects and the role of parental knowledge," Developmental Psychology, vol. 48, no. 6, pp. 1540-1552, 2012, doi: 10.1037/a0027720.

[4] E. Sikkens, M. van San, S. Sieckelinck, and M. de Winter, "Parents' Perspectives on Radicalization: A Qualitative Study," Journal of Child and Family Studies, vol. 27, no. 7, pp. 2276-2284, 2018, doi: 10.1007/s10826-018-1048-x.

[5] L. Gjersing, J. R. Caplehorn, and T. Clausen, "Cross-cultural adaptation of research instruments: Language, setting, time and statistical considerations," BMC Medical Research Methodology, vol. 10, 2010, doi: 10.1186/1471-2288-10-13.

[6] C. J. Gaskin and B. Happell, "On exploratory factor analysis: A review of recent evidence, an assessment of current practice, and recommendations for future use," International Journal of Nursing Studies, vol. 51, no. 3. pp. 511-521, Mar. 2014. doi: 10.1016/j.ijnurstu.2013.10.005.

[7] M. W. Chang, R. Brown, and S. Nitzke, "Scale development: Factors affecting diet, exercise, and stress management (FADESM)," BMC Public Health, vol. 8, no. February, 2008, doi: 10.1186/1471-2458-8-76.

[8] J. Nunnally and I. Bernstein, "Psychometric theory (3rd ed.)," Journal of APPLIED PSYCHOLOGICAL MEASUREMENT, vol. 19, no. 3, pp. 275-280, 1994, doi: 10.1177/073428299901700307.

[9] M. Tavakol and R. Dennick, "Making sense of Cronbach's alpha," International journal of medical education, vol. 2, pp. 53-55, 2011, doi: 10.5116/ijme.4dfb.8dfd.

[10] M. M. Mukaka, "Statistics Corner: A guide to appropriate use of Correlation coefficient in medical research," Malawi Medical Journal, vol. 24, no. 3, pp. 69-71, 2012, [Online]. Available: www.mmj.medcol.mw

[11] H. E. A. Tinsley and D. J. Tinsley, "Uses of Factor Analysis in Counseling Psychology Research," Journal of Counseling Psychology, vol. 34, no. 4, pp. 414-424, 1987, doi: 10.1037/0022-0167.34.4.414.

[12] J. Osborne and W. Costello, Best Practices in Exploratory Factor Analysis, no. October. 2005. doi: 10.4135/9781412995627.d8.

[13] Y. Li, "Confirmatory Factor Analysis with Continuous and Ordinal Data: An Empirical Study of Stress Level," Uppsala University, Department of Statistics, 2014

[14] L. T. Hu and P. M. Bentler, "Cutoff criteria for fit indexes in covariance structure analysis: Conventional criteria versus new alternatives," Structural Equation Modeling, vol. 6, no. 1, pp. 1-55, 1999, doi: 10.1080/10705519909540118.

[15] T. Frijns, L. Keijsers, S. Branje, and W. Meeus, "What parents don't know and how it may affect their children: Qualifying the disclosureadjustment link," Journal of Adolescence, vol. 33, no. 2, pp. 261-270, 2010, doi: 10.1016/j.adolescence.2009.05.010.

[16] S. J. Latendresse, F. Ye, T. Chung, A. Hipwell, and C. E. Sartor, "Parental Monitoring and Alcohol Use Across Adolescence in Black and White Girls: A Cross-Lagged Panel Mixture Model," Alcoholism: Clinical and Experimental Research, vol. 41, no. 6, pp. 1144-1153, 2017, doi: 10.1111/acer.13386.

[17] K. Margret, H. Stattin, and W. J. Burk, "A reinterpretation of parental monitoring in longitudinal perspective," Journal of Research on Adolescence, vol. 20, no. 1, pp. 39-64, 2010, doi: 10.1111/j.15327795.2009.00623.x.

[18] R. Rekker, L. Keijsers, S. Branje, H. Koot, and W. Meeus, "The interplay of parental monitoring and socioeconomic status in predicting minor delinquency between and within adolescents," Journal of Adolescence, vol. 59, pp. 155-165, 2017, doi: 10.1016/j.adolescence.2017.06.001.

[19] R. N. Waizenhofer, C. M. Buchanan, and J. Jackson-Newsom, "Mothers' and fathers' knowledge of adolescents' daily activities: Its sources and its links with adolescent adjustment," Journal of Family Psychology, vol. 18, no. 2, pp. 348-360, 2004, doi: 10.1037/0893-3200.18.2.348.

[20] S. Arafat, H. Chowdhury, M. Qusar, and M. Hafez, "Cross Cultural Adaptation and Psychometric Validation of Research Instruments: a
Methodological Review," Journal of Behavioral Health, vol. 5, no. 3, p. 129, 2016, doi: 10.5455/jbh.20160615121755.

[21] O. Cutrín, L. Maneiro, J. Sobral, and J. A. Gómez-Fraguela, "Longitudinal Validation of a New Measure to Assess Parental Knowledge and its Sources in Spanish Adolescents," Journal of Child and Family Studies, vol. 28, no. 5, pp. 1220-1235, 2019, doi: 10.1007/s10826-019-01366-z.

[22] F. Lionetti, L. Keijsers, A. Dellagiulia, and M. Pastore, "Evidence of factorial validity of parental knowledge, control and solicitation, and adolescent disclosure scales: When the ordered nature of Likert scales matters," Frontiers in Psychology, vol. 7, no. JUN, 2016, doi: 10.3389/fpsyg.2016.00941.

[23] S. J. Racz and R. J. McMahon, "The Relationship Between Parental Knowledge and Monitoring and Child and Adolescent Conduct Problems: A 10-Year Update," Clinical Child and Family Psychology Review, vol. 14, no. 4, pp. 377-398, 2011, doi: 10.1007/s10567-0110099-y.

[24] T. A. Schmitt, "Current methodological considerations in exploratory and confirmatory factor analysis," Journal of Psychoeducational Assessment, vol. 29, no. 4, pp. 304-321, 2011, doi: $10.1177 / 0734282911406653$.

[25] D. Stapleton, Connie, "Basic Concepts and Procedures of Confirmatory Factor Analysis," 1997

[26] D. Bacchini, M. C. Miranda, and G. Affuso, "Effects of parental monitoring and exposure to community violence on antisocial behavior and anxiety/depression among adolescents," Journal of Interpersonal Violence, vol. 26, no. 2, pp. 269-292, 2011, doi: $10.1177 / 0886260510362879$.

[27] F. Lionetti et al., "The development of parental monitoring during adolescence: A meta-analysis," European Journal of Developmental Psychology, vol. 5629, pp. 1-29, 2018, doi 10.1080/17405629.2018.1476233.

[28] S. L. Wilson, G. D. Zimet, and A. S. Knopf, "The Parental Monitoring Scale: Psychometric Properties and Implications for Use with Diverse Samples in North America," Journal of Adolescent Health, vol. 62, no. 2, pp. S57-S58, 2018, doi: 10.1016/j.jadohealth.2017.11.117.

[29] L. Keijsers and R. D. Laird, "Mother-adolescent monitoring dynamics and the legitimacy of parental authority," Journal of Adolescence, vol. 37, no. 5, pp. 515-524, 2014, doi: 10.1016/j.adolescence.2014.04.001.

[30] H. Stattin and M. Kerr, "Parental monitoring: A reinterpretation," Child Development, vol. 71, no. 4, pp. 1072-1085, 2000, doi: 10.1111/14678624.00210 .

[31] M. Kerr and H. Stattin, Parenting of adolescents: Action or reaction?, no. October. 2003. doi: 10.4324/9781410607430.

\section{AUTHORS}

First Author - Gayani K. P. De Silva, Senior Registrar - MD Community Medicine, School Health Unit, Family Health Bureau,pmkg8276@gmail.com.

Second Author - Devani S. Dissanayake, Consultant Community Physician and Senior Lecturer, Department of Community Medicine, Faculty of Medicine, University of Peradeniya, devanisaku@gmail.com.

Correspondence Author - Gayani K.P. De Silva, Senior Registrar - MD Community Medicine, School Health Unit, Family Health Bureau, pmkg8276@gmail.com, +94776109517. 